\title{
Efficiency improvement for assigning of cutting conditions on the basis of the thermo-EMF signal
}

\author{
Zhanna Tikhonova ${ }^{1, *}$, Dmitriy Kraiynev ${ }^{1}$, and Evgeniy Frolov ${ }^{1}$ \\ ${ }^{1}$ Volgograde State Technical University, Linine Ave. 28, Volgograde 400005, Russia
}

\begin{abstract}
In the realities of modern machine-building production, control and management of processes occurring in the cutting zone are of great importance. The acquisition of control will significantly improve the stability and quality of processing, and the possibility of monitoring will help to increase the economic efficiency of production. This article presents the potential of the thermo-emf signal in a natural thermocouple "tool - workpiece" which is considered to be an information signal from the cutting zone. It also shows experimental proof of the influence between honing width on the front surface of the tool and its size.
\end{abstract}

Complex automation of production processes is the main direction of modern machinebuilding development within the framework of the global concept "Industry 4.0" and a $\mathrm{CNC}$ machine is its main subject.

The lack of a reliable solution to the problem of selecting main parameters for the metalworking process remains one of the most important tasks connected with the above mentioned process, in spite of the increased capabilities of computing and control technology, including modern PC-NC system with a huge amount of memory. These parameters include the choice of the optimum cutting speed, meant to provide the required period of tool life and processing capacity. They also involve determining the optimum value of the cutting force components and this parameter in return identifies the power characteristics of cutting, the amount of torque, the calculated values for the gripping force of the workpiece in the device, expected geometric deviation of the workpiece shape, estimation of the calculated value of the quality parameters in the treated surface, especially roughness. The problem lies not in the capabilities of control technology, but in the adequacy of mathematical models of the parameters in the cutting process, which form the foundation of their algorithms for computerized analysis.

V. K. Starkov [1] put forward the concept of ensuring the stability and quality of the metalworking process by improving mathematical models for calculating its parameters due to obtaining preliminary information about the properties of each tool and a machined workpiece.

In the development of this concept, a fundamentally new method for calculating the parameters while turning and milling processes of steels was proposed in the works $[2,3$,

* Corresponding author: tihonova-ganna@rambler.ru 
4]. It is based on obtaining preliminary information about the properties of contact pairs due to the thermo-electromotive force (thermo-emf) which always accompanies edge cutting machining by tool material and it is generated by a natural thermocouple "tool workpiece". The variation of thermo-emf signal is known to occur due to a change in the work function of electrons from the hard alloy and the machined workpiece, and the value of the contact component of the thermo-emf signal in the "tool - workpiece" thermocouple will be determined if the electrochemical potential of electrons is equalized. This circumstance makes it possible to use the thermo-emf signal of a contact pair "tool workpiece", obtained in strictly defined test run modes, $(\mathrm{V}=100 \mathrm{~m} / \mathrm{min}, \mathrm{s}=0.1 \mathrm{~m} / \mathrm{rev}, \mathrm{t}$ $=1 \mathrm{~mm}$ ) [5], the same for all groups of machined and tool materials, as a diagnostic parameter for evaluating the physical and mechanical properties of various combinations of contact pairs "tool - workpiece", for the subsequent application and optimization of cutting modes for machining the same workpiece by this tool.

In general, the thermo-emf value of the natural thermocouple is determined by the expression [6].

$$
E=\left(\alpha_{2}-\alpha_{1}\right)\left(\Theta_{a}-\Theta_{b}\right)=\alpha_{1-2}\left(\Theta_{a}-\Theta_{b}\right)
$$

where $\alpha_{1}$ and $\alpha_{2}$ are specific or differential thermo-emf values for two different materials (a tool and a steel workpiece); $\alpha_{1-2}=\alpha_{2}-\alpha_{1}$ is a specific or differential thermoemf for a given pair, depending on the nature of contacted bodies and the temperature; $\Theta_{a}$ and $\Theta_{b}$ are steady-state temperatures of hot and cold junctions of a natural thermocouple.

It should be noted that the value of the thermo-emf in a trial passage is linearly connected with the geometric parameters of the instrument and takes these changes into account promptly [6].

Replaceable faceted inserts of different shapes with chip grooves in accordance with GOST 19042-80 belong to the group of cutters with a reduced face. When their thermo-emf changes, in order to reveal the relationship of the carbon content in the alloy, it is necessary to take into account the variations in the heat exchange conditions associated with the artificial change in the size of the contact areas along the front and back faces of the tool. [7]. Replaceable faceted disposable inserts of the same brand and geometry, usually have the same angles $y$ and $y_{1}, \alpha, \gamma, \lambda$. The spread in their values from batch to batch is in the range of 20-30. This allows us to say that the heat release conditions associated with these parameters can be assumed to be the same. According to GOST 19042-80 the permissible spread in values for the width of the cutter grinding land is within the range of $0.1-0.3 \mathrm{~mm}$. But the operating practice shows that the actual dimension for the chamfer of cutter grinder of disposable inserts along the front face has significantly larger variations, namely 0.2-0.6 $\mathrm{mm}$. This circumstance leads to inhomogeneous heat release conditions during the cutting process, which directly entails a violation of the linear dependence of the thermo-emf from the carbon content in the alloy. It happens due to the non-observance of the conditions for the identical heating of the hot junction of the thermocouple. Figure 1 shows the dependence of the influence of the honing width(the contact length Wh) along the front surface on the magnitude of the thermo-emf signal of a natural thermocouple for carbide tools of square shape of grades: VK8 and T14K8 GOST 19042-80 when processing steel 45 . 


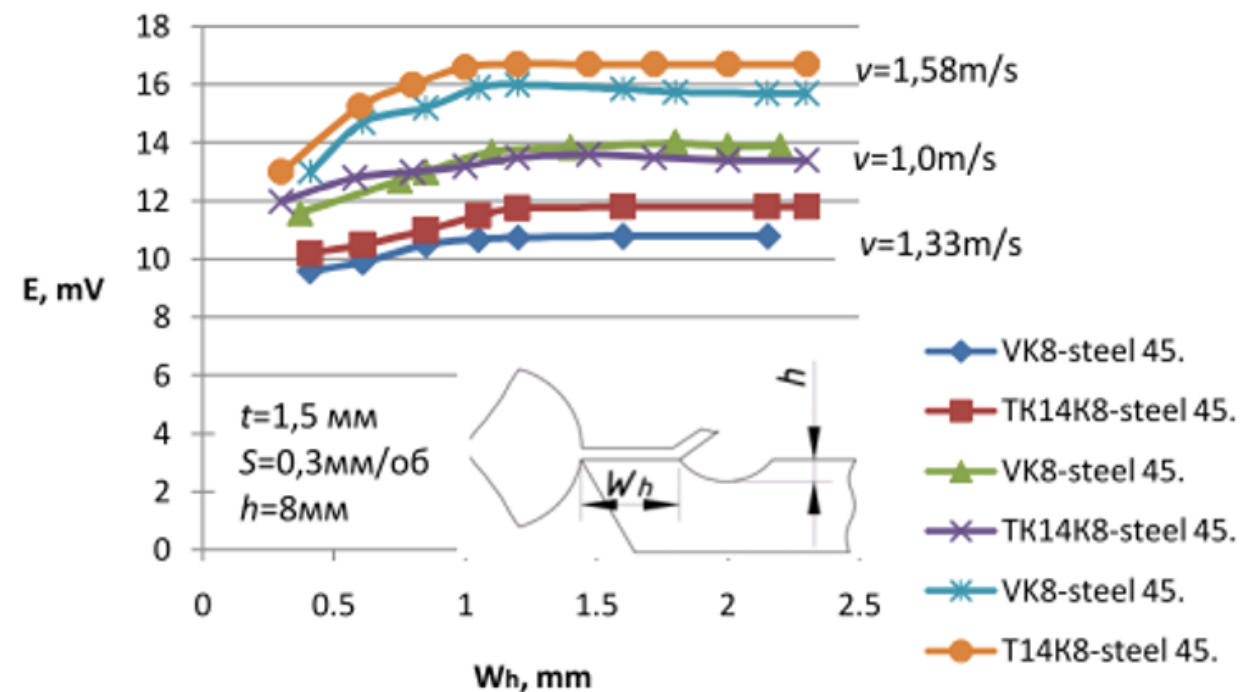

Fig. 1. Influence of the honing width Wh along the front surface of the tool on the magnitude of the thermo-emf for alloys VK8 and T14K8.

During the experiment, the heat release and heat transfer conditions for all the test inserts were identical, since the experimental sample inserts had the same geometric shape. Therefore, when the thermo-emf of the inserts 01-02 GOST 25395-90 was changed, the length of the natural contact of the chip scrap along the front surface of the tool was not violated.

The experiment started with the full length of natural contact with a smooth front surface of the insert $(\mathrm{Wh}=2.2 \ldots 2.4 \mathrm{~mm})$. Then, the length of the contact was artificially reduced by increasing the width of the chip groove at a constant depth $h$ and fixing the thermo-emf signal of the natural thermocouple after the beginning of cutting. And the value of the flank wear land in each experiment was in the range of 0.08-0.1 mm. It should be noted that the shortening of the length of full contact along the front surface to a value of $\mathrm{Wh} \sim 0.5$ has an insignificant effect (practically no effect) on the change in the thermo-emf signal of the alloys both at a speed of $1 \mathrm{~m} / \mathrm{s}$, and at a speed of $1.57 \mathrm{~m} / \mathrm{s}$. However, in the range of variation of the contact length from 1 to $0.2 \mathrm{~mm}$, a significant drop in the thermoemf signal is observed. (by $2.5-3 \mathrm{mV}$ ). This circumstance can be explained by the reduction of heat emission along the front surface of the tool, and consequently by the reduction of the heating temperature of the entire wedge. In the context of the same cutting conditions and with the same chemical composition of the carbide tool, a reduction in the contact length along the front surface by $0.1 \mathrm{~mm}$ results in a change in the thermoelectric signal of the natural thermocouple thermo-emf in the average by $0.4-0.6 \mathrm{mV}$.

Thus, the issue of preliminary evaluation of the contact pair (tool and steel workpiece) with the help of a thermo-emf signal is of a fundamental nature from the point of view of the information reliability about the cutting properties of the tool and acquires special importance for ensuring the reliability of the computer-assisted determination of edge cutting machining modes. 


\section{References}

1. Starkov V. K., Physics and optimization of metals (Mechanical engineering, Moscow, 2009)

2. Plotnikov, A.L., Sergeyev A.S., Uvarova T.V. Automated ways of determining the parameter values of turning and milling processes on CNC machines (Thin science based technologies (TNT), Volgograd, Staryy Oskol, 2017)

3. Frolov, Ye. M. Development of a mathematical model for the automated calculation of the cutting speed for thin-turning conditions, Proc. Of VSTU v. 8 pp. 108-110 (2009)

4. Frolov Ye. M., Plotnikov, A. L. Optimization of cutting modes of three stages of turning, Certificate of the state registration of the software program № 2009615674, 12.10.09 RF. VSTU, 2009.

5. Plotnikov, A.L., Sergeyev A.S.,.Zaytseva N.G; Improving the reliability of roughness control of the machined parts surface in the CAD of turning and milling operations (ZAO «ONIKS» Irbit, Tol'yatti Volgograd, 2015)

6. Yepifanov, G. I. Solid State Physics: tutorial (High School, Moscow, 1977)

7. Reznikov A.N. Thermophysics of the processes of mechanical metal-working (Mechanical engineering, Moscow, 1981) 